\title{
A class of problems that can be solved using interval algorithms
}

\author{
Bartłomiej Jacek Kubica
}

Received: 5 March 2011 / Accepted: 10 November 2011 / Published online: 23 November 2011

(C) The Author(s) 2011. This article is published with open access at Springerlink.com

\begin{abstract}
The paper discusses several theoretical and implementational problems of interval branch-and-bound methods. A trial to define a class of problems that can be solved with such methods is done. Features and variants of the method are presented. Useful data structures and shared-memory parallelization issues are considered.
\end{abstract}

Keywords Interval methods · Branch-and-bound · Predicate logic · Data structures · Multithreaded programming

Mathematics Subject Classification (2000) 03B35 Most controversial · 65G40 Certainly · 65Y05 Almost certainly · 68N17 Probably

\section{Introduction}

Interval methods $[6,8,9]$ are commonly used to solve problems like nonlinear equations or global optimization. Also other, more sophisticated problems, like the approximation of multicriterion problem's Pareto set $[4,13,18,28]$ or seeking Nash equilibria of a non-cooperative game $[11,19]$ have been solved using this class of algorithms.

The paper tries to investigate what kind of problems interval methods are good at solving and how can these problems be solved.

The author has presented the results of this paper during the SCAN 2010 conference in Lyon, September 2010.

B. J. Kubica $(\varangle)$

Institute of Control and Computation Engineering, WUT,

ul. Nowowiejska 15/19, 00-665 Warsaw, Poland

e-mail: bkubica@elka.pw.edu.pl 


\section{Problem formulation}

Let us consider the following generic problem (notation from [10] is adopted):

Find all $x \in \mathbb{R}^{n}$ such that $P(x)$ is fulfilled,

where $P(x)$ is a formula with a free variable $x$. Considering $x$ from spaces other than $\mathbb{R}^{n}$ is also possible [15], but will not be discussed here. We shall distinguish "variables" -free variables of $P(\cdot)$ and "parameters" - bound variables. In particular, the set of variables may be empty-we only want to verify if a sentence is true, e.g. "check if $(\forall t \in[1,4])\left(t^{2}-2 t+7 \geq 0\right)$ ".

It is worth noting that a similar understanding of the very idea of interval computations can be found in the papers of Shary (e.g. [26,27]). However, as Shary, as other papers that the author is aware of (see in particular [22] and references therein) consider only problems where sets of variables and parameters are disjoint, i.e. they do not range through the domain of the same function a few times. So they consider problems like e.g. "find all $x \in[0,4]$ such that $(\forall a \in[1,2])(a x \leq 0)$ ", but not "find all $x \in[0,4]$ such that $(\exists t \in[0,4])(f(x) \leq f(t))$ ".

On the other hand, many authors apply interval methods to problems like global optimization (e.g. [6,9]) or Pareto-front seeking (e.g. [4,13]), which can be described by this sort of formulae (see below). The purpose of this paper is to develop a general view on formulating these sort of problems and designing interval methods for solving them.

Please note also that Problem (1) can have some variants:

- find one point $x$, fulfilling $P(x)$,

- find an interval vector $\mathbf{x}$ such that all $x \in \mathbf{x}$ fulfill $P(x)$.

Such problems are intensively studied e.g. for linear systems of equations with interval parameters (see [23,24]); in this paper we consider Problem (1) in its original form, only.

Discussion of computability of Problem (1) can be found in [11]. The problem of solving interval linear systems is often formulated in the above way (e.g. [1,23,24]), but so can several other well-known problems. In particular:

- a system of (nonlinear) equations: $\{x \in X \mid h(x)=0\}$,

- a constraint satisfaction problem (CSP) with inequality constraints: $\{x \in X \mid$ $f(x) \in[\underline{y}, \bar{y}]\}$

- global optimization: $\{x \in X \mid(\forall t \in X)(f(x) \leq f(t))\}$,

- seeking Pareto set of a multicriterion problem: $\{x \in X \mid(\forall t \in X)((\forall i=$ $1, \ldots, N)\left(f_{i}(x) \leq f_{i}(t)\right)$ or $\left.\left.(\exists j \in[1 . . N])\left(f_{j}(x)<f_{j}(t)\right)\right)\right\}$,

- seeking Nash equilibria of a non-cooperative game: $\left\{x \in X \mid(\forall i=1, \ldots, N)\left(\forall t_{i} \in\right.\right.$ $\left.\left.\mathbf{x}_{i} \subseteq \mathbb{R}^{k_{i}}\right)\left(f_{i}\left(x_{\backslash i}, t_{i}\right) \geq f_{i}(x)\right)\right\}$.

There is a variety of books and papers about solving such problems using interval methods: [4-6,8,9,11-19,28], etc.

Some other, less commonly studied, examples include:

- seeking all optima of a function-local and global ones (studied e.g. for a very specific case in [29]): $\{x \in X \mid(\exists \varepsilon>0)((\forall t \in X)$ and $(d(x, t)<\varepsilon)(f(x) \leq$ $f(t)))\}$, 
- seeking local (but non-global) minimizers of a function: $\{x \in X \mid(\exists \varepsilon>0)((\forall t \in$ $X)$ and $(d(x, t)<\varepsilon)(f(x) \leq f(t)))$ and $((\exists s \in X)(f(s)<f(x)))\}$,

- seeking local minimizers of a function, for which the optima have a sufficiently large "attraction basin", e.g. containing at least an interval of the given range: $\{x \in X \mid(\exists \varepsilon>\delta)((\forall t \in X)$ and $(d(x, t)<\varepsilon))(f(x) \leq f(t))\}$,

- and many other similar problems.

The quantity $d(\cdot, \cdot)$, used in above formulae, denotes the metric.

All of these problems can be solved using the so-called branch-and-bound procedure.

\section{The generic algorithm}

Well-known interval algorithms for global optimization are called "branch-andbound" methods (e.g. [9]). Algorithms for seeking solutions of nonlinear equations systems or CSPs are often called "branch-and-prune" [5]. A general name, sometimes encountered, is "branch-and- $\varphi$ ", but—as it is very rarely encountered-we shall not adopt it. The term we are going to use is the "branch-and-bound method", but we understand it in a very broad sense, applying to several kinds of problems. Such algorithms can be described by the following pseudocode:

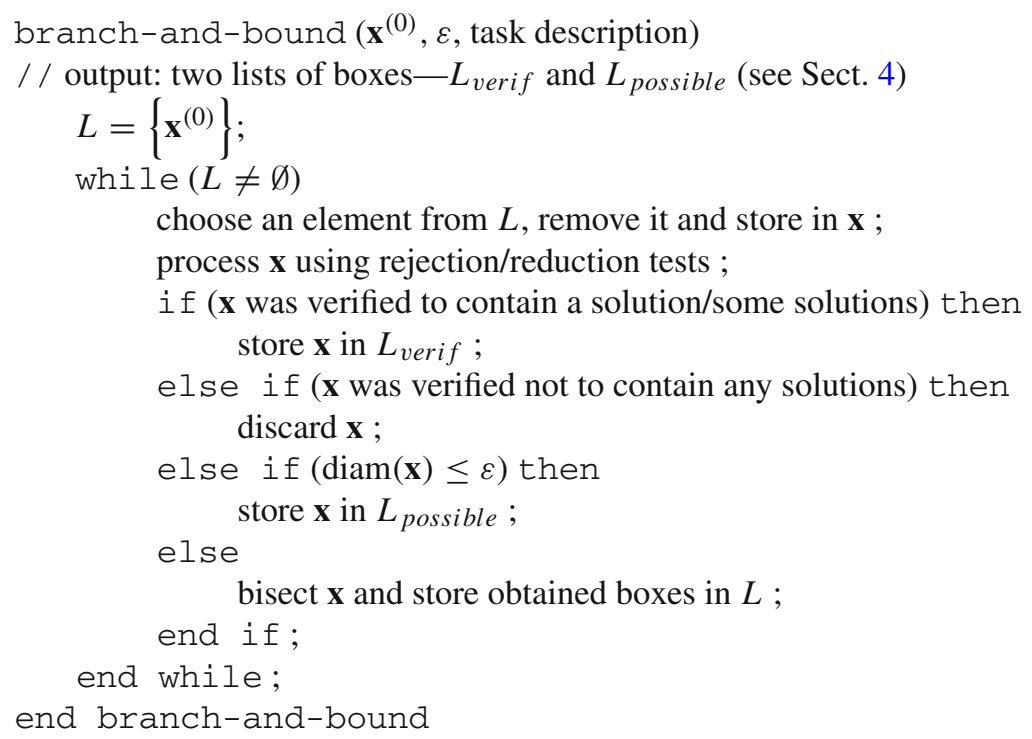

For several problems we are only able to verify some necessary condition for solutions. So boxes obtained on both lists after termination of the branch-and-bound procedure are only "suspicious" for containing actual solutions. This is the case e.g. for global optimization, when we can verify boxes to be critical points (e.g. fulfill F. John optimality necessary conditions), but do not know a priori if they are global or local minimizers. For seeking Pareto or Nash points the situation is analogous. In such 
cases, it is useful to execute a "second phase" after termination of the branch-andbound procedure. In this phase we try to delete boxes that do not contain solutions (e.g. the lower bound for the objective is too high-in global optimization).

The above generic branch-and-bound schema does not specify several significant details:

- what are the rejection/reduction tests,

- how can we verify existence of solution(s) in $\mathbf{x}$ and what exactly can we verify,

- is there any other information (but $L$ ) stored and used by the tests,

- how is the list (or other data structure!) $L$ organized,

- how and in what order are boxes chosen from $L$.

All these details depend on the problem under solution, strongly. As the rejection/reduction tests we can use a great deal of tools, developed by interval analysis. In particular these are:

- several kinds of interval Newton operator, e.g. [6,9],

- checking bounds on function values, constraints, etc.,

- checking bounds on derivatives (e.g. the monotonicity test for global optimization, concavity test, etc.),

- several constraint propagation methods, e.g. [8,5,22],

$-\ldots$

Which of the tools should be used and in what way, depends on the problem (e.g. Newton operators applied to systems of equations seek their zeros, but applied to global optimization seek stationary points of the gradients or of the F. John conditions).

It is worth noting that the Newton operators allow us not only to discard the boxes (or their parts) that do not contain solutions. These operators, under suitable circumstances, give us the possibility to verify that a box contains some solutions (see e.g. $[6,9,12])$.

Other details of specific implementations of the algorithm are going to be considered in subsequent sections.

\section{The solution set}

What can the interval branch-and-bound procedure compute? Usually, two lists of boxes are constructed-and also the pseudocode from Sect. 3 does that:

- verified solutions-boxes that are guaranteed to contain some points within the solution set,

- possible solutions-boxes that (under the given accuracy) could not be verified either to contain solutions or not to contain them.

It is problem-dependent, what the list of verified boxes contains precisely. It is related to the geometry of the solution set. And the solution set can have three forms:

- a countable set (a set of isolated points) - then verified solutions are boxes guaranteed to contain a single solution, 
- an uncountable set with an empty interior - then verified solutions are boxes guaranteed to contain a segment of the solution set,

- an uncountable set with a nonempty interior-then verified solutions are boxes guaranteed to lie in the interior of the solution set, i.e. to contain only actual solutions.

Please note that each of the above possibilities is quite often encountered in practical problems:

- a countable set is the solution to (non-singular) global optimization problems and well-determined nonlinear equations systems,

- an uncountable set with an empty interior is the solution to Pareto set seeking problem and underdetermined systems of equations,

- an uncountable set with a nonempty interior is often the solution to constraint satisfaction problems with inequality constraints.

Remark It seems tempting to develop a general interval branch-and-bound algorithm that would be able to localize all kinds of solutions. Indeed, all semialgebraic sets (and probably most other sets, but for algebraic relations we would obtain a semialgebraic solution set-see [1]) can be represented as a union of three sets: set of isolated solutions, an interior-less set of uncountably many solutions and a continuous set with a nonempty interior. Unfortunately, it seems extremely difficult to discover the type of solution if we do not know a priori what we seek.

Consider for example solving an underdetermined system of nonlinear equations. We have $m$ equations in $n$ variables $(m<n)$ and a box $\mathbf{x}$. As proved in [12], if there is a set $J$ of $m$ variable indexes such that the results of Newton operator on $x$ computations $x_{j}, j \in J$ will give results from the interior of $\mathbf{x}_{j}$ then:

$$
(\forall k \notin J)\left(\forall x \in \mathbf{x}_{k}\right)(\forall j \in J)\left(\exists ! x_{j} \in \mathbf{x}_{j}\right)\left(f\left(x_{1}, \ldots, x_{n}\right)=0\right) .
$$

If we know the number $m$ of equations, we can simply use the above property to enclose segments of the solution set.

But what about the case when we solve the equations system:

$$
\begin{aligned}
& \left(x_{1}^{2}+x_{2}^{2}-1\right) \cdot\left(x_{1}+x_{2}\right)=0 \\
& \left(x_{1}^{2}+x_{2}^{2}-1\right) \cdot\left(x_{1}-x_{2}\right)=0
\end{aligned}
$$

the solution is the union of a circle and the point $(0,0)$. But can the circle be verified using the theorem from [12]? It seems extremely difficult to distinguish between System (3) and the following one:

$$
\begin{aligned}
& \left(x_{1}^{2}+x_{2}^{2}-1+\varepsilon_{1}\right) \cdot\left(x_{1}+x_{2}\right)=0 \\
& \left(x_{1}^{2}+x_{2}^{2}-1+\varepsilon_{2}\right) \cdot\left(x_{1}-x_{2}\right)=0
\end{aligned}
$$

where $\varepsilon_{1}$ and $\varepsilon_{2}$ are close, but different. And System (4) has only one solution-the point $(0,0)$. 
Consequently, known applications of interval Newton operators (and all other tools) allow to verify at most the solution $(0,0)$; other solutions will be covered by boxes from the "possible" list.

\section{Additional information stored by the branch-and-bound method}

For equations systems and CSPs (at least unquantified - see [22]), no additional information is needed. The existence of a solution in one box is unrelated to its existence in another one.

Property 1 If the formula $P(x)$ does not contain any quantifiers ranging over $X$ then no "global" information needs to be stored either for the efficiency or for correctness.

Please note that the converse property is not fulfilled: for some problems $P(x)$ may contain some quantifiers ranging over the domain, but all the information is local and nothing but boxes has to be stored (e.g. seeking local optima).

Algorithms for global optimization are more demanding - an upper bound for the global minimum should be stored [9] so that boxes with too high lower bound on the objective could be deleted.

For the Pareto set computations we have to store yet more information. Upper bounds on criteria values for several Pareto points will be useful [20].

Yet more sophisticated is the Nash points computation [19]. Here we have to store upper bounds on players' cost functions in specific areas of the decision space.

In this case (and probably for several other ones) the data structure called an interval tree [3] may become very useful. This classical data structure has been of surprisingly rare use by the interval community up to now. The only known paper to suggest its use is [19].

\section{The order of boxes to be considered}

The order of boxes to consider is another important feature, distinguishing different versions of the branch-and-bound method. This order is related to the information that can be obtained from the boxes (see Sect. 5).

When solving equations systems or unquantified CSPs, existence of solutions in one box is not related to its existence in another one and the order of boxes is usually irrelevant. In this case we can use any structure to store boxes to be considered-a stack, a queue, a pool, etc. Also parallelization is simple, as discussed in Sect. 8.

For global optimization we have a different situation. While processing subsequent boxes we compute an upper bound on the global minimum; this minimum is used to discard boxes not containing the global minimizer. Consequently, the order of boxes is crucial for efficiency - the boxes should be ordered according to increasing lower bound on function values (e.g. [6,9]).

For computing the Pareto set or Nash equilibria seeking the order of boxes also matters, but the relation is rather complicated. Consequently, using any order seems reasonable (as for equations systems), though developing some clever heuristics might be worthwhile. 


\section{Variables versus parameters}

Yet one implementation detail should be considered as it can have a great influence on the solver's efficiency. The question is: what parameters (in the sense defined in Sect. 2) should we use?

It might seem, the number of parameters and their meaning is determined by the formulation of the problem under solution, but there can be several equivalent formulations. As a simple example, let us consider global optimization. Instead of formulation from Sect. 2:

$$
\{x \in X \mid(\forall t \in X)(f(x) \leq f(t))\},
$$

we can use an equivalent, but different one:

$$
\{x \in X \mid(\exists y=f(x))(\forall t \in X)(y \leq f(t))\},
$$

The problems are equivalent, but the branch-and-bound procedure bisecting an additional parameter and using additional rejection/reduction tests (e.g. checking consistency for $f(x)-y=0$ can perform quite differently from the version not doing it.

For global optimization such an algorithm has been proposed by Shary [25] and an analogous one for Pareto set seeking-by Kubica and Woźniak [13,14,20]. Experiments in [20] have shown a notable efficiency of this approach.

This observation seems particularly important, as proper formulation of the problem can hardly be done automatically. This means that, as we can give several general rules on using the branch-and-bound type methods, it would be very difficult to implement a generic and efficient version, using e.g. template meta-programming. But a human can formulate an efficient version of the method for a specific problem.

\section{Parallelization}

Several tools can be used to parallelize interval computations. In particular, sharedmemory parallelization has already been done using OpenMP [2], POSIX threads [14], Boost threads [16], Intel Threading Building Blocks (TBB) [17].

Parallelization of the branch-and-bound algorithm is of particular interest as:

- multicore architectures are very common nowadays,

- the structure of both branch-and-bound procedure and the "second phase" allows easy parallelization (different boxes can be processed in parallel).

As both phases of the algorithm can be parallelized, they cannot be classified as "embarrassingly parallel". At least two issues can occur:

- storing the additional information that has to be shared between different threads (tasks, processes, etc.),

- adding new boxes on the run after bisection (requires synchronization and/or smart representation of the list $L$ ). 
As we can expect, parallelizing solvers of equations systems or CSPs, is the simplest. Only the set $L$ of boxes has to be stored (no other shared data, that require synchronization) and the order of processing boxes is not significant, so any data structure - a stack, a queue, a pool [21] of boxes is sufficient. There are relatively efficient concurrent implementations of such structures (see e.g. [21]) and for some frameworks, like Intel TBB [7] one does not even have to represent the box list explicitly-it can be represented by an internal, highly efficient task list. See e.g. $[16,17]$ for details.

Multithreaded implementations of other branch-and-bound type methods are more challenging as they require to share a more sophisticated data structure: a priority queue (global optimization), a binary search tree (Pareto set approximation) or even an interval tree (Nash equilibria seeking). As e.g. vectors, queues or hash tables have several efficient, highly tuned, concurrent implementations (some of them are included in the TBB library [7]; see also [21]), it is not the case for structures we consider. (It is worth noting that recently, on 23rd of December 2010, in TBB 3.0 update 4, a concurrent priority queue has been added, as a "Community Preview" feature. But efficient implementations are yet to be determined.) A further research in this area would be very useful for concurrent interval methods.

\section{Relation to results of other researchers}

As already mentioned, this research is related to the ones of Shary [24,26,27], yet more general as it is not restricted to linear systems.

Also, considerations and results presented in this paper can be considered complementary to the investigations of Ratschan (e.g. [22]), who developed narrowing operators to achieve "first-order consistency" for arbitrary quantified CSPs.

His research is purely theoretical and addresses few (or even none) problems related to actual implementation or efficiency. In particular, his operators can be applied to a specific quantified CSP that is equivalent to a global optimization problem-like (5) or (6) - but it would have to traverse the function domain at least twice (for $x$ and $t$ ), while well-known interval branch-and-bound methods do it only once, storing the information about upper bound on the global minimum.

\section{Conclusions}

Interval branch-and-bound methods can be (and already are) used to solve a large variety of problems - the ones described by (1). Several general rules and advices can be given for implementation of such solvers, but many details are highly problem-dependent. A generic implementation (based e.g. on template meta-programming) seems rather hopeless. Yet, efficient implementations - even multithreaded ones-can be developed, but they require good understanding of the problem under solution and using knowledge about different topics-concurrent data structures (to store boxes or/and other shared data), mathematical analysis, topology, etc. (to develop efficient rejection/reduction tests), possibly also first-order (or even higher order) logic and/or quantifier elimination (to formulate the problem in a proper way). 
Acknowledgments The author would hereby like to acknowledge his discussions with Adam Woźniak. Thanks to the anonymous referees for helpful comments.

Open Access This article is distributed under the terms of the Creative Commons Attribution Noncommercial License which permits any noncommercial use, distribution, and reproduction in any medium, provided the original author(s) and source are credited.

\section{References}

1. Alefeld G, Kreinovich V, Mayer G (1998) The shape of the solution set for systems of interval linear equations with dependent coefficients. Math Nachr 192:23-36

2. Beelitz T, Lang B, Bischof CH (2006) Efficient task scheduling in the parallel result-verifying solution of nonlinear systems. Reliab Comput 12:141-151

3. Cormen TH, Leiserson CE, Rivest RL, Stein C (2009) Introduction to algorithms. MIT Press, Cambridge

4. Fernandez J, Toth B (2007) Obtaining an outer approximation of the efficient set of nonlinear biobjective problems. J Glob Optim 38:315-331

5. Goualard F (2005) On considering an interval constraint solving algorithm as a free-steering nonlinear Gauss-Seidel procedure. In: SAC'05 proceedings of the 2005 ACM symposium on applied computing, ACM, New York

6. Hansen E, Walster W (2004) Global optimization using interval analysis. Marcel Dekker, New York

7. Intel threading building blocks. http://www.threadingbuildingblocks.org

8. Jaulin L, Kieffer M, Didrit O, Walter E (2001) Applied interval analysis. Springer, London

9. Kearfott RB (1996) Rigorous global search: continuous problems. Kluwer, Dordrecht

10. Kearfott RB, Nakao MT, Neumaier A, Rump SM, Shary SP, van Hentenryck P (2002) Standardized notation in interval analysis. http://www.mat.univie.ac.at/ neum/software/int/notation.ps.gz

11. Kreinovich V, Kubica BJ (2010) From computing sets of optima, Pareto sets and sets of Nash equilibria to general decision-related set computations. J Univers Comput Sci 16:2657-2685

12. Kubica BJ (2011) Interval methods for solving underdetermined nonlinear equations systems 2008. SCAN 2008 proceedings. Reliab Comput 15(3):207-217

13. Kubica BJ, Woźniak A (2008) Interval methods for computing the Pareto-front of a multicriterial problem. PPAM 2007 proceedings. LNCS 4967:1382-1391

14. Kubica BJ, Woźniak A (2012) A multi-threaded interval algorithm for the Pareto-front computation in a multi-core environment. Presented at PARA 2008 conference, accepted for publication in LNCS 6126

15. Kubica BJ (2008) Can interval computations be applied over spaces of non-numbers?. Prace Naukowe Politechniki Warszawskiej. Elektronika 165:103-112

16. Kubica BJ (2009) Shared-memory parallelization of an interval equations systems solver-comparison of tools. Prace Naukowe Politechniki Warszawskiej. Elektronika 169:121-128

17. Kubica BJ (2009) Intel TBB as a tool for parallelization of an interval solver of nonlinear equations systems. ICCE internal report no 09-02

18. Kubica BJ, Woźniak A (2010) Using the second-order information in Pareto-set computations of a multi-criteria problem. Presented at PARA 2010 conference, accepted for publication in LNCS

19. Kubica BJ, Woźniak A (2010) An interval method for seeking the Nash equilibria of non-cooperative games. PPAM 2009 proceedings. LNCS 6068:446-455

20. Kubica BJ, Woźniak A (2010) Computing Pareto sets of multicriteria problems using interval methods. Presented at SCAN 2010 conference

21. Moir M, Shavit N (2007) Concurrent data structures. http://www.cs.tau.ac.il/ shanir/ concurrent-data-structures.pdf

22. Ratschan S (2002) Continuous first-order constraint satisfaction. LNCS 2385:181-195

23. Sharaya IA (2001) On maximal inner estimation of the solution sets of linear systems with interval parameters. Reliab Comput 7:409-424

24. Shary SP (1996) Algebraic approach to the interval linear static identification, tolerance and control problems, or one more application of Kaucher arithmetic. Reliab Comput 2(1):3-33

25. Shary SP (2001) A surprising approach in interval global optimization. Reliab Comput 7:497-505 
26. Shary SP (2002) A new technique in systems analysis under interval uncertainty and ambiguity. Reliab Comput 8:321-418

27. Shary SP (2010) Konechnomiernyi Intiervalnyi Analiz. XYZ

28. Toth BG, Kreinovich V (2009) Verified methods for computing Pareto-sets: general algorithmic analysis. Int J Appl Math Comput Sci 19(3):369-380

29. Villaverde K, Kreinovich V (1993) A linear-time algorithm that locates local extrema of a function of one variable from interval measurement results. Interval Comput 4:176-194 